\title{
Imaging of Ocular Angle Structures with Fourier Domain Optical Coherence Tomography
}

\author{
${ }^{1}$ Sanjay Asrani, ${ }^{2}$ Mei Young, ${ }^{3}$ Jing Xu, ${ }^{4}$ Marinko V Sarunic
}

\begin{abstract}
Background: Imaging of the Schlemm's Canal is complicated by the small physiological size and the location several hundred microns beneath the sclera. Noninvasive imaging of Schlemm's canal and Trabecular Meshwork (TM) in vivo with Fourier Domain Optical Coherence Tomography (FD OCT) can provide clinicians with a powerful tool to visualize ocular angular structures crucial for glaucoma management.
\end{abstract}

Purpose: To investigate the appearance of Schlemm's canal and TM on FD OCT images.

Methods: FD OCT images of the Schlemm's canal and TM were obtained with three different wavelengths using prototype FD OCT systems in a normal volunteer. FD OCT images using the $1310 \mathrm{~nm}$ wavelength prototype were obtained in three representative cases of glaucoma surgery performed on angle structures.

Results: The longer imaging depth and deeper tissue penetration of the $1310 \mathrm{~nm}$ system provided the clearest image of the TM and Schlemm's canal in the normal patient. In case 1, images pre- and post-trabectome surgery clearly showed the location and appearance of TM. In case 2, images postcanaloplasty surgery showed the location and appearance of Schlemm's canal. In case 3, images pre- and post-trabeculotomy surgery further confirms the appearance and location of the Schlemm's canal and TM.

Conclusion: Operating wavelength of the FD OCT system and exact location of the scan across different meridians minimally affects the appearance of the ocular anatomy. The postoperative images of three angle glaucoma surgeries confirmed the location of Schlemm's canal and TM.

Keywords: Optical coherence tomography, Anterior eye segment, Trabecular meshwork.

How to cite this article: Asrani S, Young M, Xu J, Sarunic MV. Imaging of Ocular Angle Structures with Fourier Domain Optical Coherence Tomography. J Current Glau Prac 2013;7(2):85-87.

Source of support: Nil

Conflict of interest: None

\section{INTRODUCTION}

Noninvasive visualization of the Schlemm's canal and trabecular meshwork (TM) in vivo would permit improved research and assist in the clinical management of glaucoma. Imaging of the SC in particular is complicated by the small physiological size and the location several hundred microns beneath the sclera. The ability to image SC has been demonstrated using high frequency ultrasound (UBM 50 $\mathrm{MHz}),{ }^{1}$ time domain optical coherence tomography $(\mathrm{OCT})^{2}$ and more recently, swept source fourier domain (FD) OCT systems. The latter have provided higher speed and higher resolution images of the ocular angle permitting clearer visualization of the $\mathrm{SC}$ and the TM. ${ }^{3,4}$ Commercial FD OCT systems have also depicted SC (SC) and TM differently and this may lead to some confusion about the structures identified.

In this report, we depict SC and TM with three different wavelengths using prototype FD OCT systems in a normal volunteer to show the variation in the images obtained. We also depict the $\mathrm{SC}$ and TM location in a few representative cases of glaucoma surgery performed on angle structures to confirm that the structures labeled in the normal anatomy are indeed the same.

\section{MATERIALS AND METHODS}

We investigated FD OCT systems for imaging the $\mathrm{SC}$ and TM at three wavelengths commonly used in OCT: $1,310 \mathrm{~nm}$ swept source, 1,060 nm swept source and $830 \mathrm{~nm}$ spectraldomain OCT. The differences of the three systems are described in Table 1.

B-scan images of the ocular angle from same volunteer with all three systems are shown in Figures 1A to C. The same features are observed with all systems, with minor differences due to the different tissue back scattering properties of imaging wavelength.

Figure 1D is a schematic of the locations of multiple B-scans acquired on a subject's eye. The shape difference of SC at two locations is presented through comparison of Figures $1 \mathrm{E}$ and F. Despite being from the same eye and

Table 1: OCT properties operating at different wavelengths

\begin{tabular}{llll}
\hline & \multicolumn{3}{l}{ Operating wavelength } \\
\cline { 2 - 4 } Properties & $830 \mathrm{~nm}$ & $1,060 \mathrm{~nm}$ & $1,310 \mathrm{~nm}$ \\
\hline Axial resolution (tissue) & $\sim 9 \mu \mathrm{m}$ & $\sim 9 \mu \mathrm{m}$ & $\sim 3 \mu \mathrm{m}$ \\
Tissue scattering (relative) & High & Medium & Low \\
Water absorption (relative) & Low & Medium & High \\
Laser source & Super luminescent & Wavelength-swept & Wavelength-swept \\
& diode (Superlum, & laser (Micron Optics & laser (Micron Optics \\
& Moscow, Russia) & Inc., Atlanta, GA) & Inc., Atlanta, GA) \\
\hline
\end{tabular}



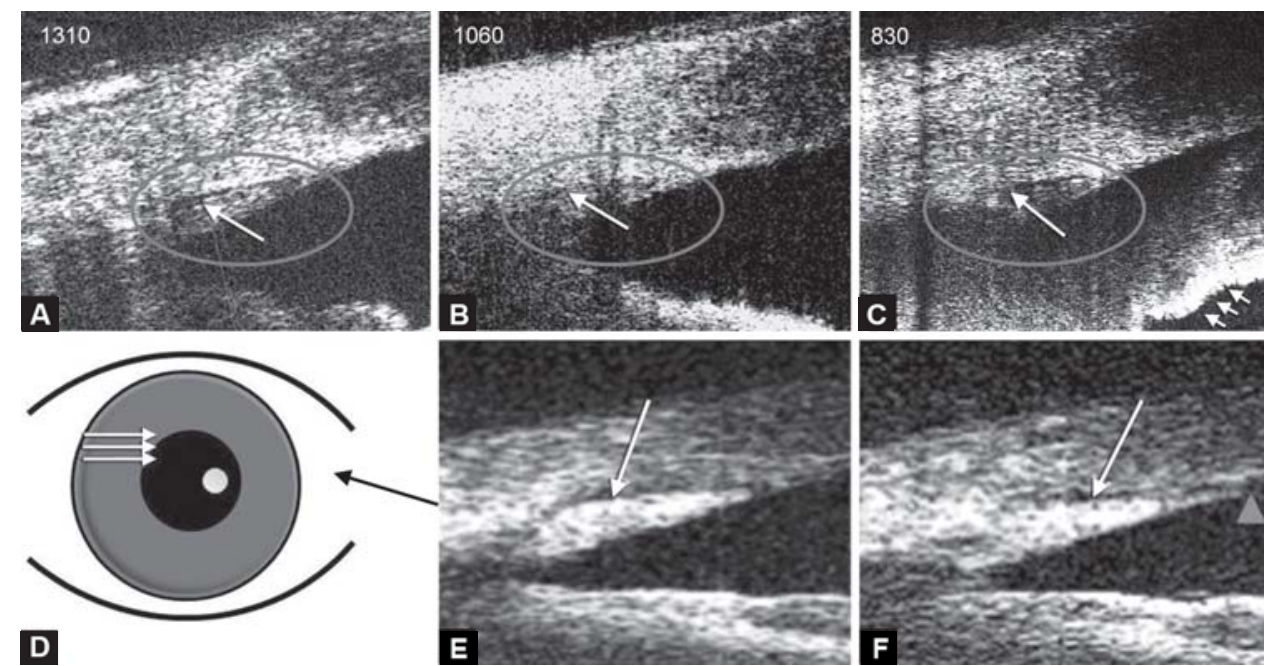

Figs 1A to F: Images of the Schlemm's canal with three different wavelengths (A to C) and with the 1,310 nm wavelength swept OCT system at different points on the subject's eye ( $D$ to F). The shapes of the regions identified as the TM and the Schlemm's canal (long arrow) changes at different points in the elevation
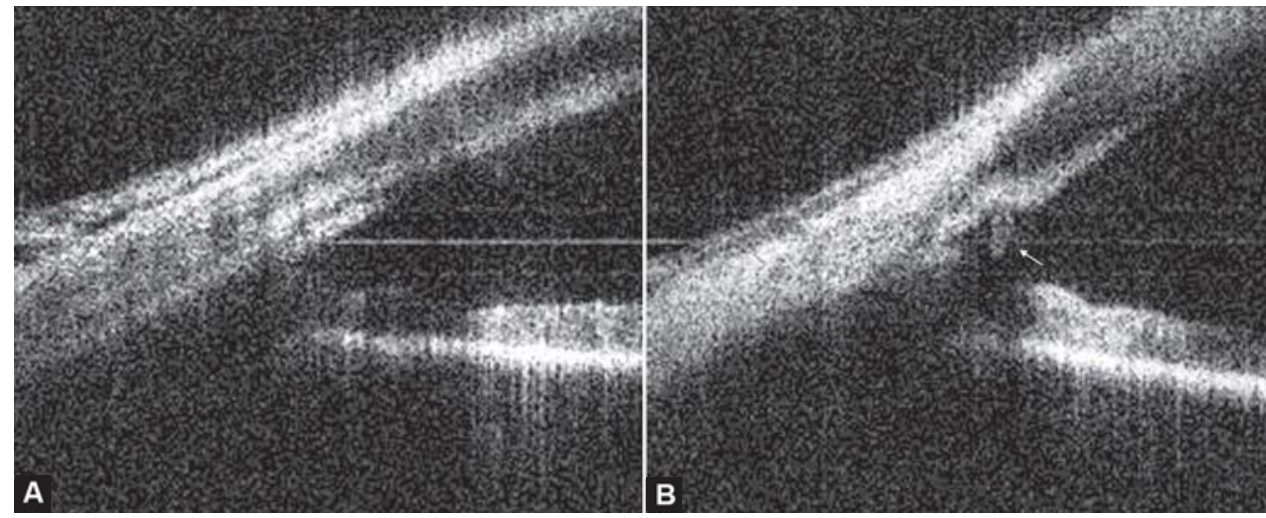

Figs 2A and B: Ocular anterior chamber angle: (A) Pretrabectome surgery and (B) post-trabectome surgery

from the same system, the shape of SC appears different in both images. In Figure 1F, the optical reflectivity of the tissue around the TM creates an artifactual appearance of the SC is communicating with the anterior chamber (triangle).

\section{CASE REPORTS}

The 1,310 nm wavelength swept FD OCT system was used to study the angle in 143 patients. The SC and TM are shown in three representative cases where surgical procedures had been performed on the angle structures.

\section{Case 1}

An elderly male with moderate glaucoma in his right eye underwent trabectome surgery. The image prior to the surgery shows the intact TM in Figure 2A. The image taken 2 days after surgery is presented in Figure 2B and shows the incision into the TM with the SC exposed to the anterior chamber with two cut edges of the meshwork on either side (arrow).

\section{Case 2}

A female in her 70's with moderate glaucoma in her left eye underwent canaloplasty surgery which involves insertion of a $10^{\prime} 0$ proline suture for $360^{\circ}$ of the canal. Figure 3 , taken 3 months after the surgery, shows the cross-section of the proline suture within the canal.

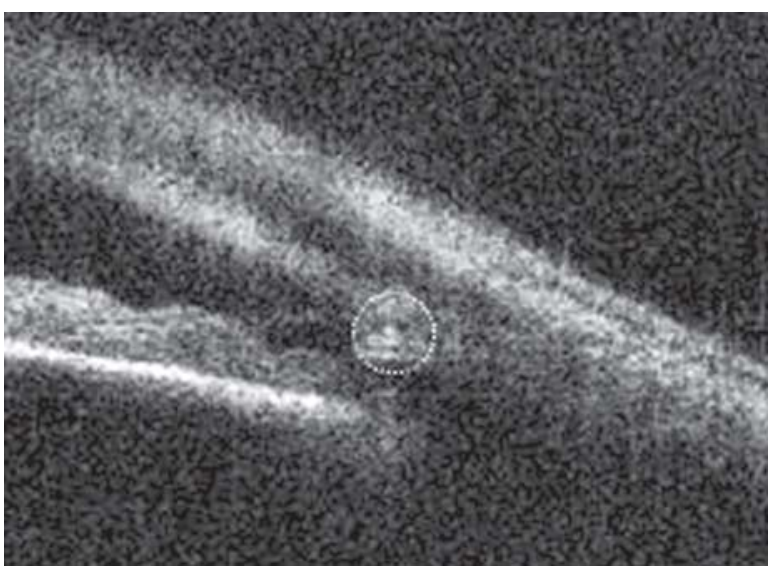

Fig. 3: Proline suture observed as a bright round reflection (in the center of the circle) within the Schlemm's canal following canaloplasty surgery 

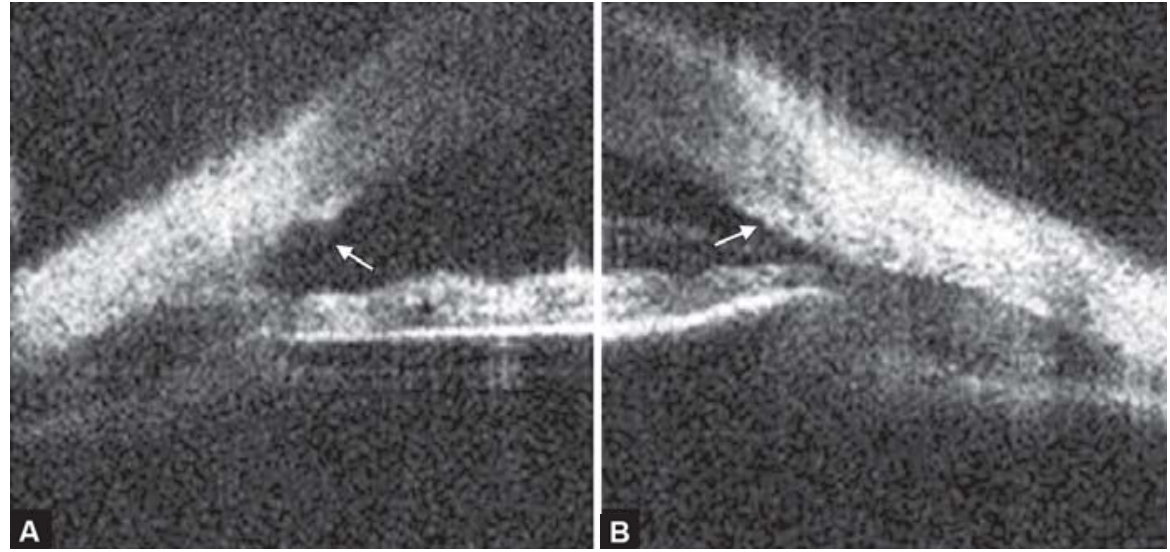

Figs 4A and B: $(A)$ The TM was surgically removed and $(B)$ the TM can be seen in the normal anatomy from the contralateral eye

\section{Case 3}

A young boy who had undergone trabeculotomy in his right eye for congenital glaucoma at age 3 . At the expected location of the SC and TM, no remnants of the TM are seen. Instead, a concave dark area is visualized Figure 4A (arrow). This results from the removed TM during the surgery and the exposed SC to the anterior chamber. In the contralateral eye, the intact TM is visualized Figure 4B.

\section{CONCLUSION}

Noninvasive imaging of the ocular angle with FD OCT permits visualization of the SC and TM. Selection of the OCT operating wavelength and exact location of the scan across different meridia minimally affects the appearance of the ocular anatomy. The postoperative images (using 1,310 nm swept source FD $\mathrm{OCT}$ ) of three angle glaucoma surgeries confirmed the location of SC and TM.

\section{ACKNOWLEDGMENTS}

We would like to thank Dr Kevin Hsu from Micron Optics for his continued support and for loaning us the prototype 1,060 and 1,310 $\mathrm{nm}$ wavelength swept laser sources.

\section{REFERENCES}

1. Irshad FA, Mayfield MS, Zurakowski D, Ayyala RS. Variation in Schlemm's canal diameter and location by ultrasound biomicroscopy. Ophthalmology 2010 May;117(5):916-920.

2. Konstantopoulos A, Hossain P, Anderson DF. Recent advances in ophthalmic anterior segment imaging: a new era for ophthalmic diagnosis? Br J Ophthalmol 2007 Apr;91(4):551-557.

3. Sarunic MV, Asrani S, Izatt JA. Imaging the ocular anterior segment with real-time, full-range Fourier-domain optical coherence tomography. Arch Ophthalmol 2008 Apr;126(4):537-542.

4. Asrani S, Sarunic M, Santiago C, Izatt J. Detailed visualization of the anterior segment using fourier-domain optical coherence tomography. Arch Ophthalmol 2008 Jun; 126(6):765-771.

${ }^{1}$ Professor, ${ }^{2}$ Biomedical Engineer

${ }^{3} \mathrm{PhD}$ Candidate, ${ }^{4}$ Associate Professor

${ }^{1}$ Department of Ophthalmology, Duke Eye Center, Duke University, Durham, North Carolina, USA

${ }^{2}$ Department of Ophthalmology and Visual Sciences, University of British Columbia, British Columbia, Canada

${ }^{3,4}$ School of Engineering Science, Simon Fraser University British Columbia, Canada

Corresponding Author: Sanjay Asrani, Professor, Department of Ophthalmology, Duke Eye Center, Duke University, Durham North Carolina, Box 3802, USA, Phone: 919-6848656, Fax: 919-6818267, e-mail: asran002@mc.duke. edu 